\title{
Method of choosing the unification of cable sections of electric network cables under conditions of load development uncertainty
}

\author{
A.D. Taslimov ${ }^{1}$, A.S. Berdishev ${ }^{2}$, M.V. Melikuziev ${ }^{1}$, F.M. Rakhimov ${ }^{2}$ \\ ${ }^{1}$ Tashkent State Technical University named after Islam Karimov, 100095, Uzbekistan, Tashkent, University St. 2A \\ ${ }^{2}$ Tashkent institute of irrigation and agricultural mechanization engineers
}

\begin{abstract}
The article analyzes the optimization criteria used in the conditions of uncertainty of the initial information, selects the number and values of standard cable sections under uncertainty, the development of the load over time using the minimax criteria of Wald and Savage, and also makes recommendations on the number and values of standard sections of distribution cables electrical networks
\end{abstract}

When predicting the optimal development and construction of distribution electric networks (DEN), one should take into account the technical and economic possibilities of choosing the optimal parameters with an increase in electrical loads over a fairly wide range. In this case, on the basis of mathematical models of DEN, the optimal network parameters are identified that correspond to the series of changes in the initial information and the optimal construction of the DEN for a number of parameters is revealed, which on a certain scale reflects the development of this network in time. The resulting sets of optimal parameters determine the rational construction of the network and the development strategy of the parameters [1].

Under these conditions, the problem of choosing the optimal parameters of the DEN is solved taking into account the uncertainty of a complex of factors characterizing the development of the load in the considered time period. Such factors are: the normative coefficient of capital investment efficiency, the initial load ratio in fractions of the project, the laws of load growth over time; annual uniform and uneven load growth; the year of reaching the design load from the moment the transformer substation (TS) was commissioned; the considered period of time, for example, the estimated life of the designed network before its reconstruction [2].

According to [3], the choice of the optimal values of the parameters of the DEN should be based on the optimization criterion in the conditions of uncertainty of the initial information. Depending on a varying degree of uncertainty, information is classified as "information situations". The information situation is the separation of the uncertainty of the states of nature from a given set $\mathrm{S}$ $=\left\{\mathrm{S}_{1}, \ldots, \mathrm{S}_{\mathrm{n}}\right\}$ of which decision-maker $(\mathrm{DM})$ has at the time of obtaining the solution.

The categories of information situations are developed and seven categories of information situations are proposed. If the information situation is characterized by a fuzzy set of states of nature, then the choice problem is solved by methods of the theory of fuzzy sets [4]. Depending on whether the information belongs to a specific information situation, it is necessary to use one or another criterion for choosing a solution that is widely used in solving practical problems $[5 ; 6]$.

When the probabilities of all values of the indefinite factor are known (the first information situation), the methods of the theory of statistical decisions are used, namely the Bayes criterion. Moreover, the optimal solution corresponds to the maximum (minimum) of the mathematical expectation of the efficiency vector, weighted by the probabilities of the state of the medium. For discrete given probabilities of states of nature, the Bayes criterion is written as follows [5]:

$$
\sum_{j=1}^{n}\left(p_{j} \cdot Y_{j k}\right) \rightarrow \min
$$

where $p_{j}$ - is the probability of the state of nature, and $\sum_{j=1}^{n} p_{j}=1$

, where $n$ - is the number of all considered states of nature; $Y_{j k}$ - is the efficiency vector (functional) for the $\kappa$-th strategy under the $j$-th state of nature.

Usually, under conditions of uncertainty in the development of the load, the distribution density $F(\beta)$ of the annual growth rate of the load $\beta$, which is regarded as a continuously distributed random variable, can be known. In the analytical representation of the dependence of the efficiency vector $\mathrm{Y}(\beta)$ on the random variable $\beta$, the Bayes criterion is written as follows [5]:

$$
\int_{q_{\min }}^{q_{\max }} F(\beta) \cdot Y(\beta) \cdot d \beta \rightarrow \min
$$

In the first and second information situations, the optimal solution is selected based on the Bayes criterion. 
In the third informational situation, the solution is chosen based on the theory of relations [3].

The Bernoulli - Laplace criterion is used to select a solution in the fourth information situation. Moreover, if there is no data on the state of nature, then it is believed that one of the states of nature is more likely than any other. This criterion is recommended as the simplest one for determining the probabilities of the state of nature. The Bernoulli - Laplace criterion is mathematically described as follows [3]:

$$
\frac{1}{n} \cdot \sum_{j=1}^{n} Y_{j k} \rightarrow \min
$$

In the fifth informational situation, with the antagonistic interests of nature, the Wald or Savage criterion is used for the solution.

According to Wald's criterion, the optimal solution is selected by the condition:

$$
\max _{j} Y_{j k} \rightarrow \min
$$

When applying the Savage criterion, the concept of risk $R_{j k}$ is used and the optimal solution is selected by the condition $[3,6]$ :

$$
R_{j k}=Y_{j k}-\min _{j} Y_{j k}
$$

According to the Savage criterion, the optimal strategy is the one in which the risk is minimal in the worst conditions [6]:

$$
\max _{j} R_{j k} \rightarrow \min
$$

A common drawback of choosing a solution for both criteria is that the solutions obtained are not very sensitive to additional information about uncertain factors.

In the sixth informational situation is the Hurwitz criterion. The condition for choosing a strategy for this criterion is [3]:

$$
\left(\xi \cdot \max _{j} Y_{j k}+(1-\xi) \cdot \min _{j} Y_{j k}\right) \rightarrow \min
$$

where $\xi$ is the pessimism coefficient chosen by the DM between zero and one.

As a result of the analysis and processing of additional information, the probabilities of a particular growth rate of the load (state of nature) were obtained, therefore, the Bayes criterion is the criterion for choosing a solution. But the question arises about the reliability of the results: there are a lot of urban DEN, they are built in a variety of conditions (the area of the city district, surface load density, consumers, urban planning requirements) and with various parameters (voltage, power, cross-section). Under these conditions, it is impossible to reliably determine the probabilities of the pace of development.

Thus, to make a more informed decision, one can propose using the following method: in any information situation from the first to the fourth (it is these information situations that characterize the state of the initial information of the technical system and, in particular, the power supply system), the decision is determined by three criteria: Walda, Savage, and Bernoulli-Laplace, which do not take into account the probability of uncertain factors, despite the fact that the criteria of Wald and Savage are the selection criteria in the fifth information situation (antagonistic nature). If the decisions obtained by these three criteria coincide, then this decision is taken as rational; if the obtained solutions do not coincide, then the rational solution is determined by the zoning method.

When applying minimax criteria, the task of choosing the optimal number of cable cross-sections is solved during the "game with nature", in which the number of cable cross-sections are strategies, and the laws of load growth, estimated period, time to reach design load, etc. - vague states of nature. Many states of nature are limited by the range of possible values of the minimum and maximum limits of change of the resulting uncertain factor.

When choosing the number of cable cross-sections, the optimization criterion is [7.8]:

$$
\begin{gathered}
Z\left(N_{F}, \Phi\right)=\delta_{1}+\delta_{2}+\delta_{3} N_{F, i}^{-1}+\delta_{4} \Phi N_{F}^{0,3} \\
N_{F} \in\{1, N\}, \quad \Phi \in S, \quad \\
S: \Phi_{\min } \subseteq \Phi \subseteq \Phi_{\max },
\end{gathered}
$$

where $\delta_{1}, \delta_{2}, \delta_{3}, \delta_{4}$ - are the constant components of the optimization criterion.

$\Phi$ - resulting uncertain factor; S - is the set of realizations of the indefinite factor corresponding to the set of combinations of "states of nature"; $N_{F}$ - is a set of strategies corresponding to the number of cable crosssections.

Wald's criterion: In this method, the optimal strategy is that for which a minimum of maximum values of the optimization criterion is achieved:

$$
\min _{N_{F} \in N} \max _{\Phi \in S} Z\left(N_{F}, \Phi\right)
$$

The above criterion focuses on the most unfavorable circumstances by an uncertain factor. In this case, it is guaranteed that the optimization criterion - for any state of nature - will not be greater than some guaranteed value.

Since, with an increase in the value of the uncertain factor, the optimization criterion increases, we have:

$$
{ }_{\Phi \in S}^{\max } Z\left(N_{F}, \Phi\right)=\delta_{1}+\delta_{2}+\delta_{3} N_{F, i}^{-1}+\delta_{4} \Phi_{\max } N_{F}^{0,3}
$$

Then the Wald criterion takes the form:

$$
N_{F}^{\min } \in N\left[\delta_{1}+\delta_{2}+\delta_{3} N_{F, i}^{-1}+\delta_{4} \Phi_{\max } N_{F}^{0,3}\right]
$$

Thus, according to the Wald criterion, the problem of choosing the optimal number of cable cross sections is reduced to determining the minimum total costs at the maximum value of the uncertain factor.

Savage criterion: The optimal strategy is the one for which the minimum risk value (R) from not being informed about the values of the resulting uncertain factor:

$$
\begin{gathered}
\min _{N_{F} \in N} \max _{\Phi \in S} R\left(N_{F}, \Phi\right) \\
\max R\left(N_{F}, \Phi\right)=\max \left[Z\left(N_{F}, \Phi\right)-\min Z\left(N_{F}, \Phi\right)\right] \\
\text { where } \\
\Phi \in S \quad \Phi \in S \quad N_{F} \in N
\end{gathered}
$$

- the maximum possible loss relative to the minimum total cost in the field of possible values $\Phi$. Expression (3) can be reduced to the form:

$$
\begin{array}{lll}
\min & \max & \max \left[Z\left(N_{F}, \Phi\right)-Z\left(\bar{N}_{F} \in N\right)\right] \\
N_{F} \in N & \bar{N}_{F} \in N \quad \Phi \in S,
\end{array}
$$


where $N_{F}$ are the values of the number of sections corresponding to the minimum of total costs at the limit values of $\Phi$.

The maximum difference in the optimization criterion when using the $N_{F}$ strategy compared to the $\bar{N}_{F}\left(\max _{\Phi} R_{N_{F}}\right)$ strategy depends on both the maximum and minimum values of $\Phi$.

Then, taking into account (1), expression (4) takes the form:

$$
\begin{array}{lll}
\min & \max & \max \left[\delta_{3}\left(N_{F}^{-1}-\bar{N}_{F}^{-1}\right)+\delta_{4}\left(N_{F}^{0.3}-\bar{N}_{F}{ }^{0.3}\right) \Phi\right] \\
N_{F} \in N & \bar{N}_{F} \in N & \Phi \in S
\end{array}
$$

Thus, in the problem of choosing the optimal number of cable cross-sections according to the Savage criterion, the method for pairwise comparison of strategies is used [7].

The numerical solution of equations (2) and (5) gives the optimal values of the number of cable cross sections DEN 0.38 and $10 \mathrm{kV}$, determined by the criteria of Wald and Savage, respectively. With the accepted initial data [2], the optimal values of the number of cable cross sections DEN determined by the Wald and Savage criteria are given in the table.

As can be seen from the table, the influence of various laws of load growth on the choice of the optimal number of cable cross-sections for DEN is not the same when evaluated according to the Wald and Savage criteria. So, according to the Wald criterion, the optimal values of the number of cable cross sections are one or two - with a uniform law of load growth (in the entire range of electrical load density). And according to the Savage criterion, the optimal values of the number of cable cross sections practically do not depend on the law of load growth.

This character of the influence of the laws of load growth is explained by the fact that in this problem the values of the cable cross-sections that are optimal according to the Wald criterion are determined by $\Phi^{\max }$ taking into account the large role of energy losses; the latter are different for different laws of load growth. According to the Savage criterion, the optimal number of cable cross-sections is determined by $\Phi^{\text {min }}$ (a small role of energy losses), the values of which are approximately the same for the considered laws of load growth (table).

Table 1. Optimal according to the criteria of Wald and Savage, the number of cross-sections of DEN cables

\begin{tabular}{|c|c|c|c|}
\hline $\begin{array}{c}\text { Load } \\
\text { growth } \\
\text { law }\end{array}$ & Criteria & $\begin{array}{c}\text { Loop network } \\
\text { diagrams }\end{array}$ & $\begin{array}{c}\text { Dual beam } \\
\text { networks }\end{array}$ \\
\cline { 3 - 4 } Even & Wald & 2 & 1 \\
\cline { 2 - 4 } & Savage & 2 & 1 \\
\hline \multirow{2}{*}{ Uneven } & Wald & 1 & 1 \\
\cline { 2 - 4 } & Savage & 2 & 1 \\
\hline
\end{tabular}

Based on the foregoing and analysis of the results by minimax criteria given in the table, the following conclusions can be drawn.

In DEN - at low electrical load densities (up to 10-12 $\mathrm{mVA} / \mathrm{km}^{2}$ ), it is optimal to use two cable sections (240 and $95 \mathrm{~mm}^{2}$ or 185 and $70 \mathrm{~mm}^{2}$ ); - high load densities (more than $10-12 \mathrm{mVA} / \mathrm{km}^{2}$ ), the use of one cable section $\left(150\right.$ or $\left.120 \mathrm{~mm}^{2}\right)$ is optimal.

Calculation of electricity losses in cable lines for real residential areas of the city due to the use of a limited number of standard cable sections. Calculations show that the use of two standard sections instead of 6-8, selected in accordance with current standards, leads to a reduction in electricity losses in cable lines by $12-15 \%$ per year. At the same time, the decrease in specific losses of electricity on average is $20 \mathrm{kWh} /$ year per $1 \mathrm{~kW}$ of load on the tires of transformer substations.

\section{References}

1. A.D. Taslimov. Mathematical models in the optimization of parameters and structures of urban electricity supply systems // Scientific and Technical Journal "Problems of Energy and Resource Saving". Tashkent, 2017. - No. 3-4. S. 62-65.

2. A.D. Taslimov. Unification of parameters of urban distribution electric networks in conditions of uncertainty: Monograph. - T .: TashGTU, 2019 .-- 148 p.

3. Trukhaev R.I. Decision making models in the face of uncertainty. - M .: Nauka, 1981. - 258 p.

4. Leshchinskaya T.B. Methods of multicriteria optimization of rural power supply systems in the conditions of uncertainty of initial information. - M .: Agroconsult, 1998 .-- 120 p.

5. Levin M.S., Leshchinskaya T.B. Methods of solution theory in problems of optimization of power supply systems. - M.: Publishing House VIESH, part 1. 2006. $130 \mathrm{p}$.

6. Ventzel E.S. Operations research. - M.: Higher School, 2001.- 208 p.

7. Taslimov A.D., Melikuziev M.V., Murodov B.K. Development of a technical and economic model for optimizing the distribution network parameters // Journal "Vestnik TashGTU". Tashkent, 2018 .-- No. 3. P. 53-57. Bereznev Yu. I. The choice of wire cross-sections of lines 0.38 and $10 \mathrm{kV}$ in conditions of uncertainty and the problem of unification of their scale // News of higher educational institutions. Energy issues: scientific, technical and industrial journal. - 2009. - No. 9/10. S. $38-40$. 